\title{
Exploring Spatial Scale Perception in Immersive Virtual Reality for Risk Assessment in Interior Design
}

$\begin{array}{ll}\begin{array}{l}\text { Jingjing Zhang } \\ \text { HIT Lab NZ } \\ \text { University of Canterbury }\end{array} & \begin{array}{l}\text { Xiaoliang Bai } \\ \text { Cyber-Physical Interaction } \\ \text { Lew Zealand }\end{array} \\ \begin{array}{l}\text { Northwestern Polytechnica } \\ \text { jingjing.zhang@ } \\ \text { pg.canterbury.ac.nz }\end{array} & \begin{array}{l}\text { Xi'an, China } \\ \text { bxl@nwpu.edu.cn }\end{array} \\ \text { Thammathip } & \text { Simon Hoermann } \\ \text { Piumsomboon } & \text { School of Product Design } \\ \text { School of Product Design } & \text { University of Canterbury } \\ \text { University of Canterbury } & \text { New Zealand } \\ \text { New Zealand } & \text { simon.hoermann@ } \\ \text { tham.piumsomboon@ } & \text { canterbury.ac.nz } \\ \text { canterbury.ac.nz } & \text { Rob Lindeman } \\ \text { Ze Dong } & \text { HIT Lab NZ } \\ \text { HIT Lab NZ } & \text { University of Canterbury } \\ \text { University of Canterbury } & \text { New Zealand } \\ \text { ze.dong@pg.canterbury.ac.nz } & \text { gogo@hitlabnz.org }\end{array}$

Permission to make digital or hard copies of part or all of this work for personal or classroom use is granted without fee provided that copies are not made or distributed for profit or commercial advantage and that copies bear this notice and the full citation on the first page. Copyrights for third-party components of this work must be honored. For all other uses, contact the Owner/Author.

CHI'20 Extended Abstracts, April 25-30, 2020, Honolulu, HI, USA (c) 2020 Copyright is held by the owner/author(s).

ACM ISBN 978-1-4503-6819-3/20/04.

https://doi.org/10.1145/3334480.3382876

\begin{abstract}
One of the utilities of Virtual Reality is to provide its users with new perspectives, which is a promising application for architectural and interior design. In this paper, we investigate the effects of varying spatial scale perception (SSP), the perception of risks, and the ability to detect them in the virtual environment. We conducted a user study where participants experienced four unique perspectives, that of a two-year-old child, an eight-year-old child, an adult and a person in a wheelchair by manipulating their virtual inter-pupillary distance and eye height. We found that varying SSP had significant impacts on the perceived level of risk, heights of the identified risk, and the number of risks discovered. The results yielded empirical evidence to support that experiencing different SSP, can potentially help identify issues during an architectural design process for various groups of users.
\end{abstract}

\section{Author Keywords}

Spatial scale perception; risk assessment; architectural; interior design; virtual reality; multi-scale virtual environment. 


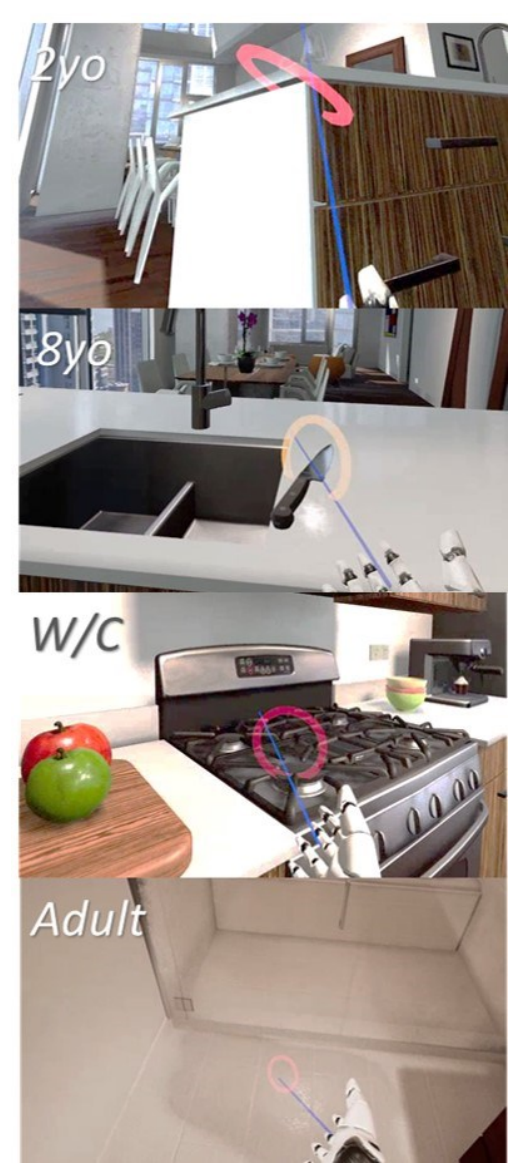

Figure 1: Spatial scale perception four perspectives, a 2-year-old child (2yo), an 8-year-old child (8yo), a person in a wheelchair $(W / C)$, and an adult (Adult).

\section{CSS Concepts}

- Human-centered computing Human computer interaction (HCI); Interaction paradigms Virtual reality; Empirical studies in $\mathrm{HCI}$;

\section{Introduction}

To create better designs, designers must empathise with their target users, who may have special needs, e.g. young children, users with disabilities. Virtual Reality (VR) has the potential to improve and accelerate the design process by letting the designers and stakeholders experience different designs from many perspectives within virtual environments (VEs). This research focuses on utilising VR for users to experience an alternate perspective in an application for interior design [4] with a focus on health and safety in household environment.

Spatial scale perception (SSP) is our ability to perceive the relative size between ourselves, objects of interest, and the surrounding environment. These experiences are subjective to each individual, and several factors influence our SSP, such as one's eye height $(\mathrm{EH})$ or inter-pupillary distance (IPD). For example, a small child with lower EH and smaller IPD would perceive the environment to be larger than an average adult [9]. Nonetheless, designers are typically adults who perceive the world from their perspective making it more challenging, not to mention other shortcomings such as a lack of standardization and regulations for the safety of children's furniture [8].

In this paper, we chose combinations of EH and IPD as our independent variables. Manipulating eye height is trivial in the real world; however, it is difficult if not impossible, to change one's IPD. However, these attributes can be trivially altered within VR. Techniques, such as spatial scale manipulation, allow users to view the world from different perspectives. Applying such a technique in a design context has the potential to help designers see the world from the target user's point of view. This can be invaluable during the design process to assist and influence the designers in their creation of better products for different groups of target users.

Past research has found that the manipulation of both EH and IPD are crucial to simulate different levels of SSP, and that altered perception could elicit different behavior from users $[9,15]$. For example, a study that let participants experience a child's perspective and virtual representation in a VE found them to illustrate child-like attributes [2]. Similarly, in another study that had the participants experience different virtual representation, one taller and the other shorter, they found that the taller ones appeared more confident during a negotiation [18].

One of the first research that demonstrated support for multiple levels of SSP for a collaborative design task was the multi-scale collaborative virtual environment or MCVE project [17]. MCVE allowed users to collaborate in the VE for an urban planning scenario. The users could view the city from a regular scale at street level or a giant scale for a better understanding of the landscape. Subsequent research also showed that providing multiple levels of SSP in a VE could improve user spatial understanding [17], enhance navigation [1, $10,15]$, and enrich collaboration [14, 16, 17]. Beyond manipulating the user's perspective, several studies investigated the effects of altering the scale of the virtual avatar's body parts such as hands [12], feet [7], self-avatar [2]. They found significant effects on the user's perception for different sizes. 

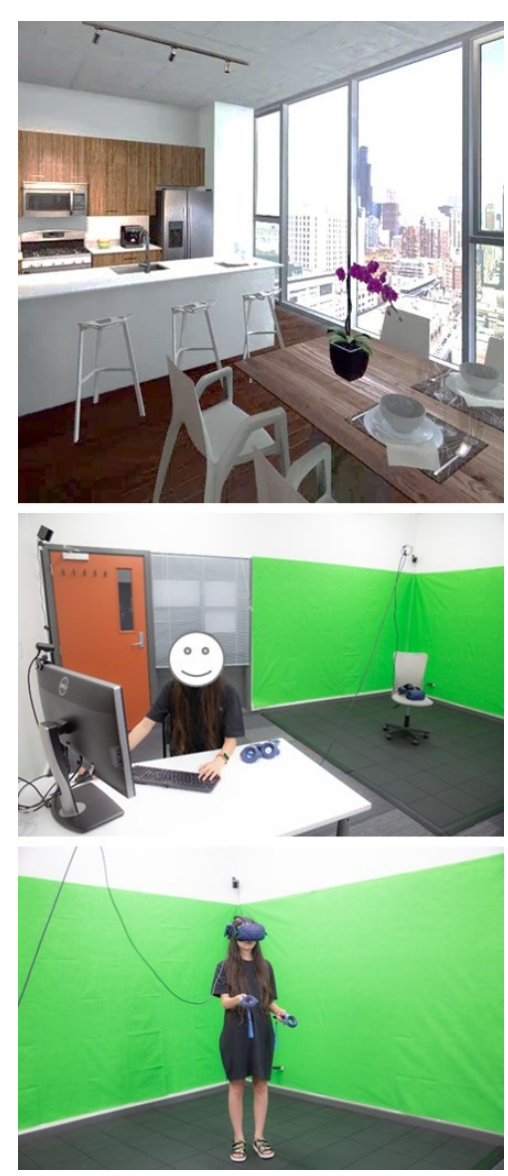

Figure 2: A virtual two-storey apartment used in the experiment (top), workstation setup and experimental space (mid), and a user in space
To the best of our knowledge, no study has investigated how the manipulation of SSP in VR can help identify potential risks to improve the safety of an interior environment, especially for children and persons with disabilities. This research has made several contributions as follows: 1 ) the first user study to investigate multiple levels of manipulation of SSP for risk assessment in interior design, 2) a VR risk assessment scenario as a tool to assist in the interior design process, 3) empirical evidence to support that the different perspectives through SSP manipulation in VR has a potential to assist designers in better design for different end-users.

\section{User Study}

We conducted a user study where the participants identified and assessed the potential risks in a virtual two-storey apartment from a different user's perspective. Our goal was to better understand the impacts of SSP manipulation in the interior architectural visualization and risk assessment task. We compared the outcomes of experiencing different perspectives, our independent variable, the user's perception of risks and hazards and their ability to identify them in the VE. Our hypotheses were:

Experiencing different perspectives in VR would significantly impact the participant's perception of risks in terms of $\boldsymbol{H 1}$ ) perceived level of risk (Risk Rating) and $\boldsymbol{H 2}$ ) the total number of risks identified (Number of Risks), and their ability to identify the risks in terms of $\boldsymbol{H 3}$ ) average risk height (Risk Height).

The Risk Rating was a 5-point Likert scale. The Risk Height was recorded in centimeters. The Number of
Risks was an accumulated number of risks identified by all seventeen participants in each condition. These were our three dependent variables. To simulate various perspectives, we manipulated three levels of $\mathrm{EH}$ and three levels of IPD as shown in Table 1. However, instead of a $3 \times 3$ factorial design between $\mathrm{EHs}$ and IPDs, we were interested in the actual perspective of different user groups and chose four perspectives to simulate (see Figure 1): a two-year-old child (2yo), eight-year-old child (8yo), an adult in a wheelchair $(W / C)$, and an adult (Adult). We based our EH selections on the growth chart published by CDC [11]. The $\mathrm{EH}$ is approximately $100 \mathrm{~mm}$ below the average height between female and male averages. The average IPD used in previous research was $63 \mathrm{~mm}[5$, 6]. However, for the child's IPD, we referred to MacLachlan and Howland [13] due to their large sample size and finer age division. The study was withinsubjects and the four conditions were counterbalanced. We recruited 17 participants from the University of Canterbury (average age of 32.4 years $(\mathrm{SD}=11.8)$, and an average height of $157.8 \mathrm{~cm}$ $(S D=41.0)$, nine females). Five participants reported

Table 1: Four conditions were chosen from different levels of manipulation of SSP between EH and IPD.

\begin{tabular}{|c|c|c|c|}
\hline IPD EH & $\begin{array}{c}\text { Low } \\
(760 \mathrm{~mm})\end{array}$ & $\begin{array}{c}\text { Moderate } \\
(1220 \mathrm{~mm})\end{array}$ & $\begin{array}{c}\text { High } \\
(1600 \mathrm{~mm})\end{array}$ \\
\hline $\begin{array}{c}\text { Small } \\
(46 \mathrm{~mm})\end{array}$ & 2 year old & & \\
\hline $\begin{array}{c}\text { Medium } \\
(54 \mathrm{~mm})\end{array}$ & & 8 year old & \\
\hline $\begin{array}{c}\text { Large } \\
(63 \mathrm{~mm})\end{array}$ & $\begin{array}{c}\text { Wheel Chair } \\
\text { (Adult) }\end{array}$ & Adult \\
\hline
\end{tabular}



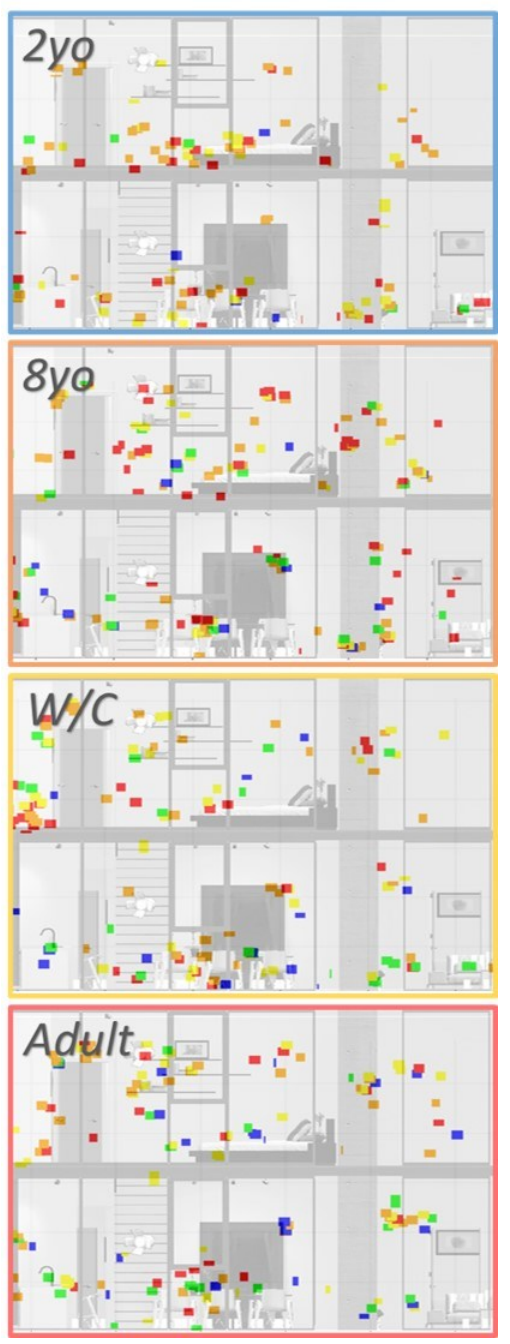

Figure 4: Partial cross sections of the apartment with 5 color dots representing all the accumulated risks rated for each condition. having children. In terms of experience with VR, seven participants had no previous VR experience, six used it a few times in a year, two monthly, one weekly, and one daily.

\section{Experimental Setup}

The design requirements for our system are 1) must support dynamic adjustments of the virtual EHs and IPDs to simulate four perspectives, 2 ) be efficient to set up and re-calibrate when required, 3) provide a realistic rendering of the $V E$, and 4) support standard navigational methods in VE. Following these design requirements, we chose a combination of hardware and software. For hardware, we used 1) HTC Vive pro VR system with one VR Headset, three Lighthouse Base Stations, and two VR Controllers, 2) A desktop PC with Intel Core i7 @ $4.40 \mathrm{GHz}, 32$ GB of RAM, and NVIDIA GeForce RTX 2080. For the software, we chose to use the Unreal Game Engine (version 4.15) to develop our system on Microsoft Windows 10 and the SteamVR API (version 1.6.10) to interface with the VR hardware. Figure 2 (top) shows a model of a virtual apartment used in our study. For the virtual representation, the participants were given only virtual hands as we wanted to keep the study simplistic and avoid any potential confounding factors. The experiment space was fully tracked with a dimension of $2.7 \times 2.7 \mathrm{~m}$ as shown in Figure 2 (mid). The participants stood in every condition except in $W / C$, where they were seated in a wheeled office chair.

\section{Procedure}

The study was approved by our university's Human Ethics Committee. Participants were given an introduction to the study, signed a consent form, and filled out a demographic questionnaire. The

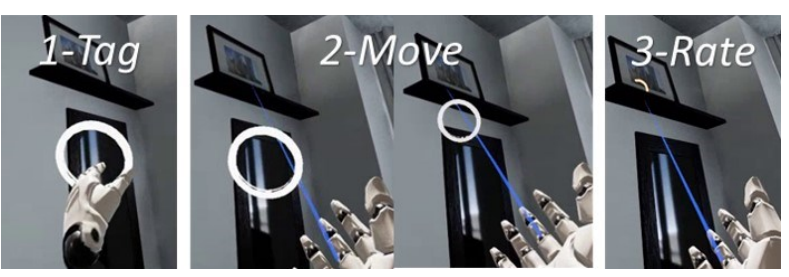

Figure 3: The risk assessment marking process comprises of 3 steps: 1) create a ring marker using a left VR

controller, 2) position the marker along a ray cast from the right VR controller, and 3) choose a color that represent 1 of 5 risk rating.

experimenter explained the risk assessment process and the types of risk that could be presented in the environment. We defined hazards as situations that pose a threat to health and safety, and risks as products of the consequence and probability of a hazardous event. The types of the risk presented were falls, fires, poisoning, drowning, cuts, and burns. Apart from the inherent risks presented in the virtual apartment such as sharp table corners and steep stairs, we included 10 randomly positioned risks to reduce the learning effects such as a knife or a flower vase.

The participants were then demonstrated how to operate the VR equipment and the VR interface in our system. They could use a touchpad on the VR controller to move forward and backward. To reduce cybersickness, they needed to physically turn with their head. Figure 3 shows the three steps for identifying and rating risks in the study, which comprises of three steps: 1) tagging (identifying the hazard and marking it), 2) moving (the hazard might be further away, so they needed to move the tag into position), and 3 ) rating (identifying the level of risk on a 5-point scale from Very Low to Very High). The rating was based on 


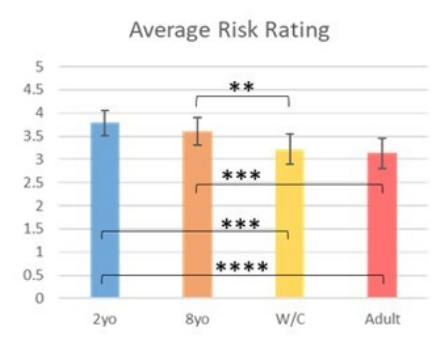

Average Risk Height
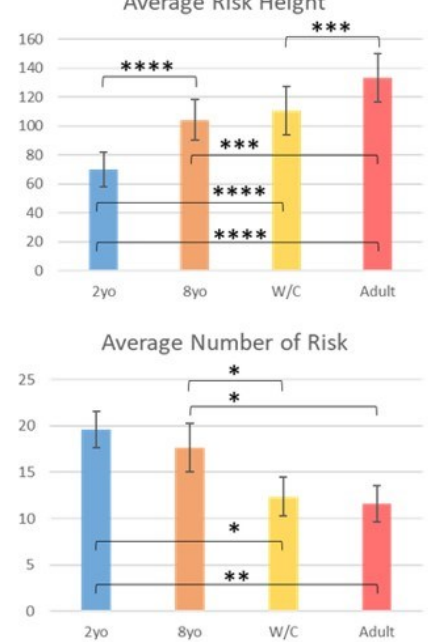

Average Session Duration (min)

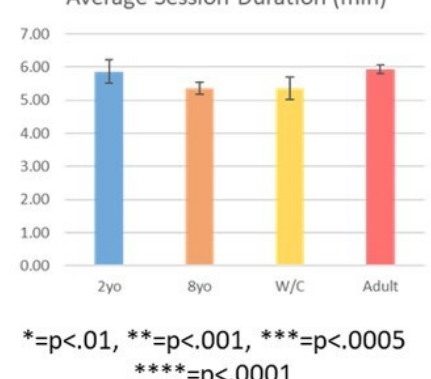

${ }^{*}=\mathrm{p}<.01,{ }^{* *}=\mathrm{p}<.001,{ }^{* * *}=\mathrm{p}<.0005$

Figure 5: Study results as plots. the perceived risk to their health and safety to themselves from their current perspective. Furthermore, the experimenter used this opportunity to calibrate the participant's virtual EH offsetting their own height such that every participant experienced the same $\mathrm{EH}$ for the same condition. The participants were then given a brief training session to familiarize themselves with our system in a different virtual room. When the actual experiment began, participants experienced four conditions in a counter-balanced order. The participants were asked to think aloud during the assessment process for the experimenter to record. As the study was completed, the participants were asked to complete a System Usability Scale (SUS) questionnaire [3]. Each session took approximately 50 minutes to complete.

Results

The Shapiro-Wilk Test indicated that our data significantly deviated from a normal distribution (Risk Rating - $\mathrm{W}=0.88, \mathrm{p}<.0001$, Risk Height - $\mathrm{W}=0.90$, $\mathrm{p}<.0001$, and Number of Risks - W=0.82, $\mathrm{p}<.0001$ ). The Friedman test yielded significant difference for Risk Rating $\quad\left(X^{2}(2)=951.9, \quad \mathrm{p}<.0001\right)$, Risk Height $\left(X^{2}(2)=1866.1, \mathrm{p}<.0001\right)$, and Number of Risks $\left(X^{2}(2)=89.0, \mathrm{p}<.0001\right)$.

For post-hoc pairwise comparisons, we used Wilcoxon signed-rank tests with Bonferroni correction ( $p$-value adjusted). Figure 5 illustrates the plots of the results. For Risk Rating, the pairwise comparisons yielded significant differences for 2yo-Adult ( $\mathrm{V}=7856.5$, $\mathrm{p}<.0001), 2 y o-W / C \quad(\mathrm{~V}=9492, \mathrm{p}<.0005), 8 y o-$ Adult $(\mathrm{V}=8384, \mathrm{p}<.0005)$ and $8 y o-W / C(\mathrm{~V}=8797, \mathrm{p}<.001)$ For Risk Height, the significant differences were found between 2yo-Adult $(\mathrm{V}=2569, \mathrm{p}<.0001), \quad 2 y o-W / C$ $(\mathrm{V}=5677, \mathrm{p}<.0001), 2$ yo-8yo $(\mathrm{V}=9709, \mathrm{p}<.0001)$,
8yo-Adult $\quad(\mathrm{V}=6860, \quad \mathrm{p}<.0005)$ and $W / C$-Adult $(\mathrm{V}=12683, \mathrm{p}<.0005)$. Lastly, for Number of Risks, the pairwise comparisons gave significant differences between 2yo-Adult $(\mathrm{V}=148, \mathrm{p}<.001), 2$ yo- $W / C(\mathrm{~V}=105$, $\mathrm{p}=.002)$, 8yo-Adult $(\mathrm{V}=120, \mathrm{p}=.008)$ and 8 yo-W/C $(\mathrm{V}=138, \mathrm{p}=.004)$. There were no significant differences for Session Duration between conditions.

\section{Discussion}

Our results provided strong evidence to support all three hypotheses, $\mathrm{H} 1, \mathrm{H} 2$, and $\mathrm{H} 3$ that experiencing different perspectives in VR would significantly impact the participant's perception of risks in terms of perceived level of risk (Risk Rating) and a total number of risk identified (Number of Risks), and their ability to identify the risks in terms of average risk height (Risk Height).

In terms of perceived level of risk or Average Risk Rating, we found that a combination of lower $\mathrm{EH}$ and smaller IPD influenced the participants judgement of perceived level of risk with the average ratings of $2 y o$ $(\bar{x}=3.8, S D=1.1), 8$ yo $(\bar{x}=3.6, S E=1.2), W / C \quad(\bar{x}=3.2$, $\mathrm{SD}=1.3)$, and Adult $(\overline{\mathrm{x}}=3.1, \mathrm{SD}=1.3)$. Participants found the risks more threatening from a child's perspective. This can be seen in Figure 4 where the level of risk and colors are from very low in blue, low in green, moderate in yellow, high in orange, and very high in red. It is evident that there is a higher density of blue and green dots in the Adult and $W / C$ conditions, and more orange and red in $2 y o$ and $8 y o$.

In terms of number of risks identified, the Average Number of Risk were $2 y o(\bar{x}=19.6, S D=8.0)$, 8yo $(\overline{\mathrm{x}}=17.7, \mathrm{SD}=10.8), W / C(\overline{\mathrm{x}}=12.4, \mathrm{SD}=8.6)$, and Adult $(\bar{x}=11.6, S D=8.0)$. There were significantly more risks found as a child in the $2 y o$ and $8 y o$ conditions 


\section{Number of Risk at Various} Height Interval

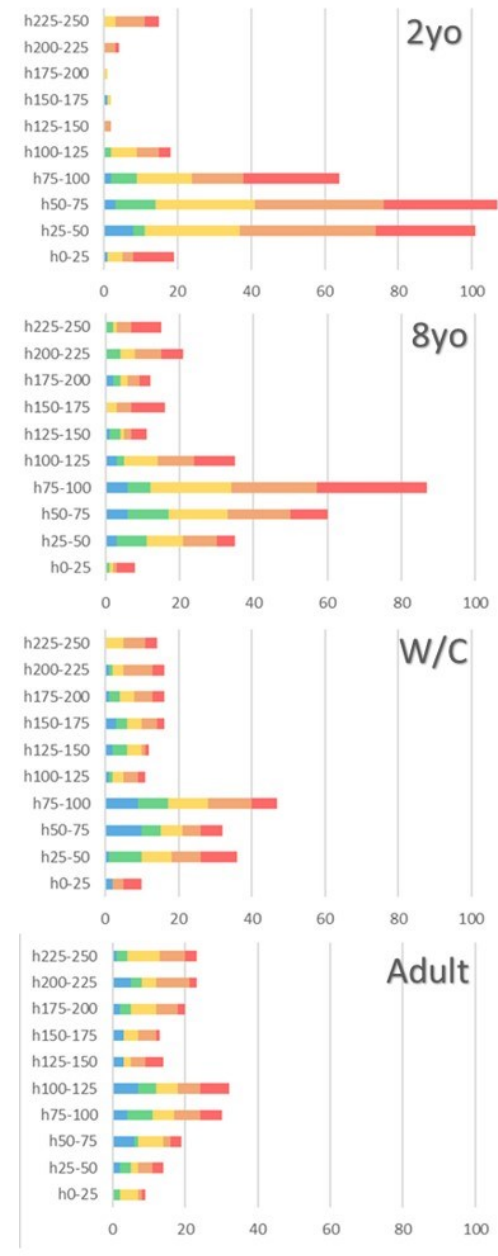

= Very Low $=$ Low $=$ Moderate $=$ High $\mid$ Very High

Figure 6: Plots of number of risks recorded for all height intervals. compared to an adult in $W / C$ and Adult. Figure 6 shows the number of risks found for various intervals of height relative to the floor level where the participants stood. We observe that taking a child's perspective made it easier for the participants to identify more risks in the VE given a similar amount of time in each condition. We found the participant's ability to identify the risks, in terms of Average Risk Height for 2 yo ( $\bar{x}=70.0$, $\mathrm{SD}=48.8)$ yielded a low height level, 8yo $(\overline{\mathrm{x}}=104.2$, $\mathrm{SD}=58.6)$ and $W / C \quad(\bar{x}=110.4, \quad S D=69.2)$ were moderate, and Adult $(\bar{x}=133.2, S D=69.8)$ produced a high level. This was our expectation that a unique level of height would provide a unique perspective and influence the average number of risks found.

From observation, we found that participants showed particular interest when they experienced a 2 yo perspective. Some participants performed child-like behaviors such as jumping, tiptoeing, or stretching their arms trying to reach difficult places. This aligns with the findings from past research [2]. We also observed that having a child's perspective made it easier for the participants to compare their size to the environment (e.g., furniture, gaps), even though, we did not provide a full-body virtual representation. We also observed that most participants were able to identify structural risks easily, e.g. sharp corners, when experiencing different perspectives. However, for more subtle risks, such as chemicals, the participants who had children were more likely to notice. In another case, participants in the $W / C$ condition found it difficult to turn around and navigate in the chair, for example in the small corridor in the VE.

With the post-experimental questionnaire, we asked the participants two questions, "Was there any benefit in experiencing different perspectives in the task?", and
"Did experiencing different perspectives influence your decision in the task?". For the first question, all participants gave a positive response and for the second, only one participant gave a neutral response. For example, P1 stated, "I didn't notice the hazards and they didn't appear to be dangerous until I saw them from another perspective". Some participants mentioned that seeing from another perspective would help them understand the others and gain insights into the needs and threats corresponding to a different age, height, and mobility. Some participants also pointed out that experiencing different perspectives in VE might be useful in other domains such as designing a playpen for children. P5 suggested that "People can try it in VR as a trial system before implementing any project". P6 said that "With this kind of system, designers can eliminate the potential hazards in the environment for different people".

\section{Conclusion and Future Work}

We present the first user study to investigate multiple levels of manipulation of spatial scale perception using VR for risk assessment in interior design. Four unique perspectives of a two-year-old child, eight-year-old child, an adult in a wheelchair, and an adult were compared in terms of the perceived level of risk, the number of risks identified, and average risk height. The results were significant and provide strong evidence that experiencing different perspectives in VR does impact the participant's perception and their ability to identify risks. For future work, we would like to further identify the types of risks that are more effective to visualize and identify through the manipulation of spatial scaled perception and also investigate the impacts of spatial scale perception on scale estimation when designing products for different target users. 


\section{Acknowledgement}

This work is supported

by National Natura

Science Foundation of

China (NSFC), Grant No: 61850410532

\section{References}

[1] Parastoo Abtahi, Mar Gonzalez-Franco, Eyal Ofek, and Anthony Steed. 2019. I'm a Giant: Walking in Large Virtual Environments at High Speed Gains. In Proceedings of the $2019 \mathrm{CHI}$ Conference on Human Factors in Computing Systems ACM, 522. https://doi.org/10.1145/3290605.3300752

[2] Domna Banakou, Raphaela Groten, and Mel Slater. 2013. Illusory ownership of a virtual child body causes overestimation of object sizes and implicit attitude changes. Proceedings of the National Academy of Sciences 110, 31, 1284612851. https://doi.org/10.1073/pnas. 1306779110

[3] John Brooke. 1996. SUS-A quick and dirty usability scale. Usability evaluation in industry 189, 194, 4-7.

[4] Özgen O. Demirbaş. 2001. The relation of learning styles and performance scores of the students in interior architecture education Bilkent University.

[5] Neil A. Dodgson. 2004. Variation and extrema of human interpupillary distance. In Stereoscopic Displays and Virtual Reality Systems XI International Society for Optics and Photonics, 3646. https://doi.org/10.1117/12.529999

[6] Tatjana Filipović. 2003. Changes in the interpupillary distance (IPD) with ages and its effect on the near convergence/distance (NC/D) ratio. Collegium antropologicum 27, 2, 723-727.

[7] Eunice Jun, Jeanine K. Stefanucci, Sarah H. Creem-Regehr, Michael N. Geuss, and William B. Thompson. 2015. Big Foot: Using the Size of a Virtual Foot to Scale Gap Width. ACM Trans. Appl. Percept. 12, 4, 1-12. http://dx.doi.org/10.1145/2811266

[8] Youjiin Jurng, and Su-Jeong Hwang. 2010. Policy Standards for Children's Furniture in

Environmental Design. Policy \& The Environment, 68
[9] Jangyoon Kim, and Victoria Interrante. 2017. Dwarf or giant: the influence of interpupillary distance and eye height on size perception in virtual environments. In Proceedings of the Proceedings of the 27th International Conference on Artificial Reality and Telexistence and 22nd Eurographics Symposium on Virtual Environments (Adelaide, Australia2017), Eurographics Association, 3298859, 153-160. http://dx.doi.org/10.2312/egve.20171353

[10] Andrey Krekhov, Sebastian Cmentowski, Katharina Emmerich, Maic Masuch, and Jens Krüger. 2018. GulliVR: A walking-oriented technique for navigation in virtual reality games based on virtual body resizing. In Proceedings of the 2018 Annual Symposium on Computer-Human Interaction in Play ACM, 243-256.

https://doi.org/10.1145/3242671.3242704

[11] Robert J. Kuczmarski. 2000. CDC growth charts; United States.

[12] Sally A. Linkenauger, Markus Leyrer, Heinrich $H$. Bülthoff, and Betty J. Mohler. 2013. Welcome to wonderland: The influence of the size and shape of a virtual hand on the perceived size and shape of virtual objects. PloS one 8, 7, e68594. https://doi.org/10.1371/journal.pone.0068594

[13] Colleen MacLachlan, and Howard C. Howland. 2002. Normal values and standard deviations for pupil diameter and interpupillary distance in subjects aged 1 month to 19 years. Ophthalmic and Physiological Optics 22, 3, 175-182. https://doi.org/10.1046/j.14751313.2002.00023.x

[14] Thammathip Piumsomboon, Arindam Day, Barrett Ens, Youngho Lee, Gun Lee, and Mark Billinghurst. 2017. Exploring enhancements for remote mixed reality collaboration. In SIGGRAPH Asia 2017 Mobile Graphics \& Interactive Applications ACM, 16. https://doi.org/10.1145/3132787.3139200 
[15] Thammathip Piumsomboon, Gun A. Lee, Barrett Ens, Bruce $\mathrm{H}$. Thomas, and Mark Billinghurst. 2018. Superman vs giant: a study on spatial perception for a multi-scale mixed reality flying telepresence interface. IEEE transactions on visualization and computer graphics 24, 11, 29742982.

https://doi.org/10.1109/TVCG.2018.2868594

[16] Thammathip Piumsomboon, Gun A. Lee, Jonathon D. Hart, Barrett Ens, Robert W. Lindeman, Bruce $\mathrm{H}$. Thomas, and Mark Billinghurst. 2018. Mini-me: an adaptive avatar for mixed reality remote collaboration. In Proceedings of the $2018 \mathrm{CHI}$ conference on human factors in computing systems ACM, 46.

https://doi.org/10.1145/3173574.3173620

[17] Zhang Xiaolong, and George W. Furnas. 2005. mCVEs: Using Cross-Scale Collaboration to Support User Interaction with Multiscale Structures. Presence: Teleoperators \& Virtual Environments 14, 1, 31-46.

http://dx.doi.org/10.1162/1054746053890288

[18] Nick Yee, and Jeremy Bailenson. 2007. The Proteus effect: The effect of transformed selfrepresentation on behavior. Human communication research 33, 3, 271-290.

https://doi.org/10.1111/j.1468-

2958.2007.00299.x 